\title{
PERFORMANCE COMPARISON DCM VERSUS QPSK FOR HIGH DATA RATES IN THE MBOFDM UWB SYSTEM
}

\author{
Naziha NOURI ${ }^{1}$, Asma MESSAOUDI ${ }^{2}$ and Ridha BOUALLEGUE ${ }^{3}$ \\ RL Innov'Com : Innovation of communicating and cooperative mobile, \\ Higher School of Communications of Tunis, Sup'Com Tunisia \\ ${ }^{1}$ nourinazihadyahoo.fr \\ ${ }^{2}$ Asma.Messaoudidenit.rnu.tn \\ ${ }^{3}$ ridha.boualleguedsupcom.rnu.tn
}

\begin{abstract}
This paper presents the advantage of using a new modulation scheme called dual carrier Modulation (DCM) compared to classical Quadrature Phase Shift Keying (QPSK) modulation. This comparison is done at data transmission broadband in Multiband OFDM system (MBOFDM) based on Ultra Wide Band UWB. Simulation results show that the use of the modulation DCM for high data rates is more efficient compared with QPSK.
\end{abstract}

\section{KEYWORDS}

ECMA368, MBOFDM, WPAN, QPSK, DCM.

\section{INTRODUCTION}

UWB communication, accepted by the Federal Communications Commission (FCC) [1] in 2002, is characterized by its low cost for high data rates over wireless personal area networks WPAN. Recently, the UWB system based on MB-OFDM has been exploited in many fields such as wireless personal area networks offering broadband over $480 \mathrm{Mbps}$, low power multimedia capabilities for PCs, user electronics, mobile and automotive market [2].

In the MB-OFDM physical layer, DCM has been suggested as a new modulation scheme for high data rates [3].

QPSK and DCM are exploited as modulation schemes for MB-OFDM in ECMA-368. QPSK constellation is used for data rates $200 \mathrm{Mb} / \mathrm{s}$ and lower, while DCM is used as a multidimensional constellation for data rates $320 \mathrm{Mb} / \mathrm{s}$ and higher.

This paper compares the performance between DCM and QPSK modulation for high rates within the ECMA-368 standard using saleh and vanzuella channel.

Natarajan Meghanathan et al. (Eds) : NETCOM, NCS, WiMoNe, GRAPH-HOC, SPM, CSEIT - 2016

pp. 73- 81, 2016. (C) CS \& IT-CSCP 2016

DOI : $10.5121 /$ csit.2016.61507 
The second section presents the studied system in general, the third one describes the two modulations QPSK and DCM, and then a performance comparison is discussed in section IV, and finally a conclusion in last Section.

\section{MB-OFDM SYSTEM ARCHITECTURE}

The architecture of the MB-OFDM transmitter is shown in Figure 1. An error correction code (FEC (Forward Error Correction)) is then applied to provide resilience against transmission errors. The punching step is essential to get high data rates.

The encoded sequence is interleaved in three consecutive steps as will be explained later, followed by the binary coding operation or symbol mapping of an OFDM symbol.

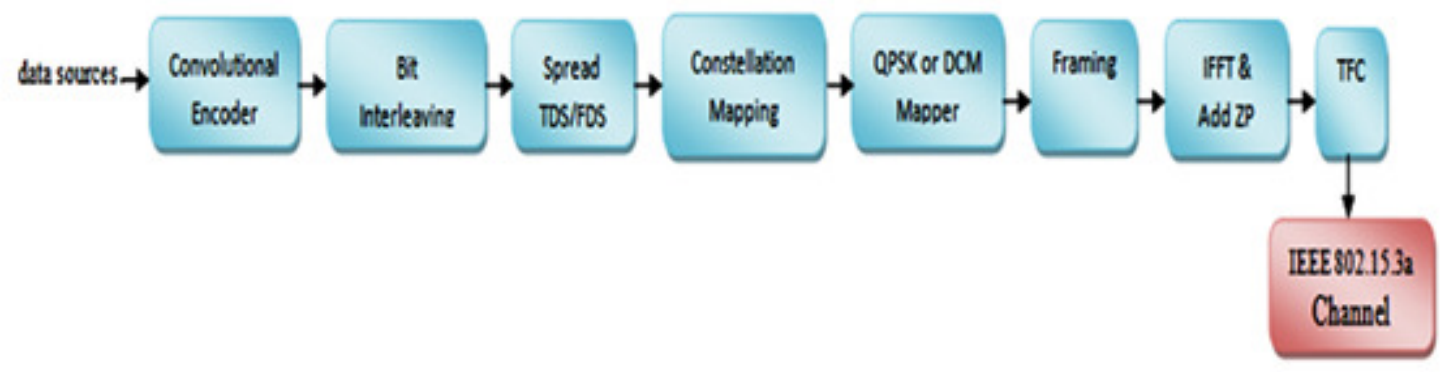

Figure 1: The transmitter architecture for the MB-OFDM system

The MB-OFDM system rates are summarized in Table 1 as they were defined in the ECMA-368 standard [4].

Tableau 1: Characteristics rates of MB-OFDM solution and number of bits per block

\begin{tabular}{|c|c|c|c|c|c|c|}
\hline $\begin{array}{l}\text { Data Rate } \\
(M B / s)\end{array}$ & Modulation & Coderate $(R)$ & FDS & TDS & $\begin{array}{l}\text { Coded Bits/6 } \\
\text { OFDM } \\
\text { Symbol } \\
\left(N_{C B P 6 S}\right) \\
\end{array}$ & $\begin{array}{l}\text { Info Bits/6 } \\
\text { OFDM } \\
\text { Symbol } \\
\left(N_{I B 6 S}\right)\end{array}$ \\
\hline $\begin{array}{l}53.3 \\
\end{array}$ & QPSK & $1 / 3$ & Yes & Yes & 300 & 100 \\
\hline 80 & QPSK & $1 / 2$ & Yes & Yes & 300 & 150 \\
\hline 106.7 & QPSK & $1 / 3$ & No & Yes & 600 & 200 \\
\hline 160 & QPSK & $1 / 2$ & No & Yes & 600 & 300 \\
\hline 200 & QPSK & $5 / 8$ & No & Yes & 600 & 375 \\
\hline 320 & DCM & $1 / 2$ & No & No & 1200 & 600 \\
\hline 400 & DCM & $5 / 8$ & No & No & 1200 & 750 \\
\hline 480 & DCM & $3 / 4$ & No & No & 1200 & 900 \\
\hline
\end{tabular}

\section{QPSK AND DCM}

\subsection{QPSK}

For rates below $200 \mathrm{Mb} / \mathrm{s}$, the interleaved binary data is mapped to a constellation into QPSK Quadrature Phase. 


$$
d[k]=k_{M O D} \times[(2 \times b[2 k]-1)+j(2 \times b[2 k+1]-1)]
$$

Where $k=0,1,2, \ldots$,

$$
\text { And } k_{M O D}=1 / \sqrt{2}
$$

\subsection{DCM}

For rates of $320 \mathrm{Mb} / \mathrm{s}$ and more, not using spreading (Tab. 1), the binary data is mapped to a multidimensional constellation using a dual-carrier modulation technique (DCM). Note that the first proposals MB-OFDM for IEEE 802.15.3a, including the proposal of September 2004, only consider a QPSK constellation for all data rates [6].

Indeed, the DCM modulation has been adopted by ECMA and recently amended in [7].

Passing from a QPSK constellation to a DCM scheme, an additional form of diversity may be obtained, which leads to an improvement in the overall range of the system.

This diversity is introduced by mapping four bits on two constellations of 16QAM (Quadrature Amplitude Modulation) shown in Figure 3.

The symbols obtained are then converted into bits which are separated by at least $200 \mathrm{MHz}$ of bandwidth, which is approximately equivalent to 50 subcarriers.

The transmission of each obtained flow is carried out, respectively, by the first half and the second half of the subcarriers of the OFDM symbol. The different stages of the DCM modulation are listed as follows:

1. Binary input data coded and interleaved, $b[i]$ where $i=0,1,2, \ldots$, are divided into groups of 200 bits and converted into complex numbers 100 using a technique called double carrier modulation.

2. The 200 coded bits are grouped into 50 groups of 4 bits as shown in Table 2. Each group is represented as $(\mathrm{b}[\mathrm{g}(\mathrm{k})], \mathrm{b}[\mathrm{g}(\mathrm{k})+1], \mathrm{b}[\mathrm{g}(\mathrm{k})+50], \mathrm{b}[\mathrm{g}(\mathrm{k}) 51])$, where $\mathrm{k} \in[0,49]$ and

$$
g(k)=\left\{\begin{array}{cc}
2 k & k \in[0.24] \\
2 k+50 & k \in[25.49]
\end{array}\right.
$$

3. Each group of 4 bits $(\mathrm{b}$ [g (k)], b [g (k) +1], b [g (k) +50], b [g (k) 51]) must be mapped to a constellation four-dimensional, and converted into two complex numbers $(\mathrm{d}[\mathrm{k}], \mathrm{d}[\mathrm{k}+50])$.

4. Complex numbers must be normalized using a normalization factor :

$$
k_{M O D}=1 / \sqrt{10}
$$

The use of non-adjacent subcarriers within this transmission makes it possible to increase the reliability of these high speeds modes towards the fading effect of the transmission channel. 
The DCM technique is not applied to the low flow rates $(200 \mathrm{Mb} / \mathrm{s}$ and below) as frequency diversity is better exploited through the use of FEC codes and TDS and FDS low flow techniques. Therefore, the expected diversity gain for these flows of DCM is minimal and the added complexity for DCM is not justified.

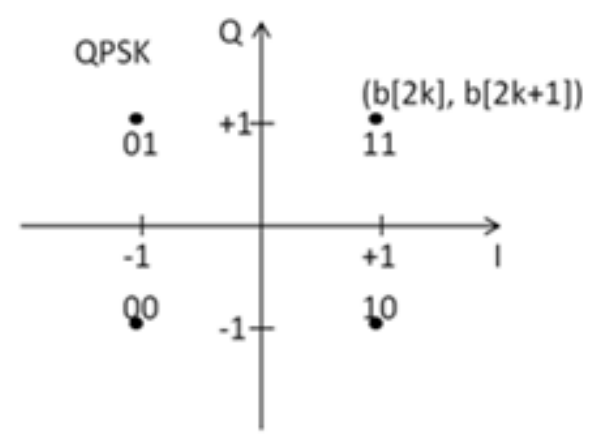

Figure 1: QPSK Constellation as standard ECM-368.

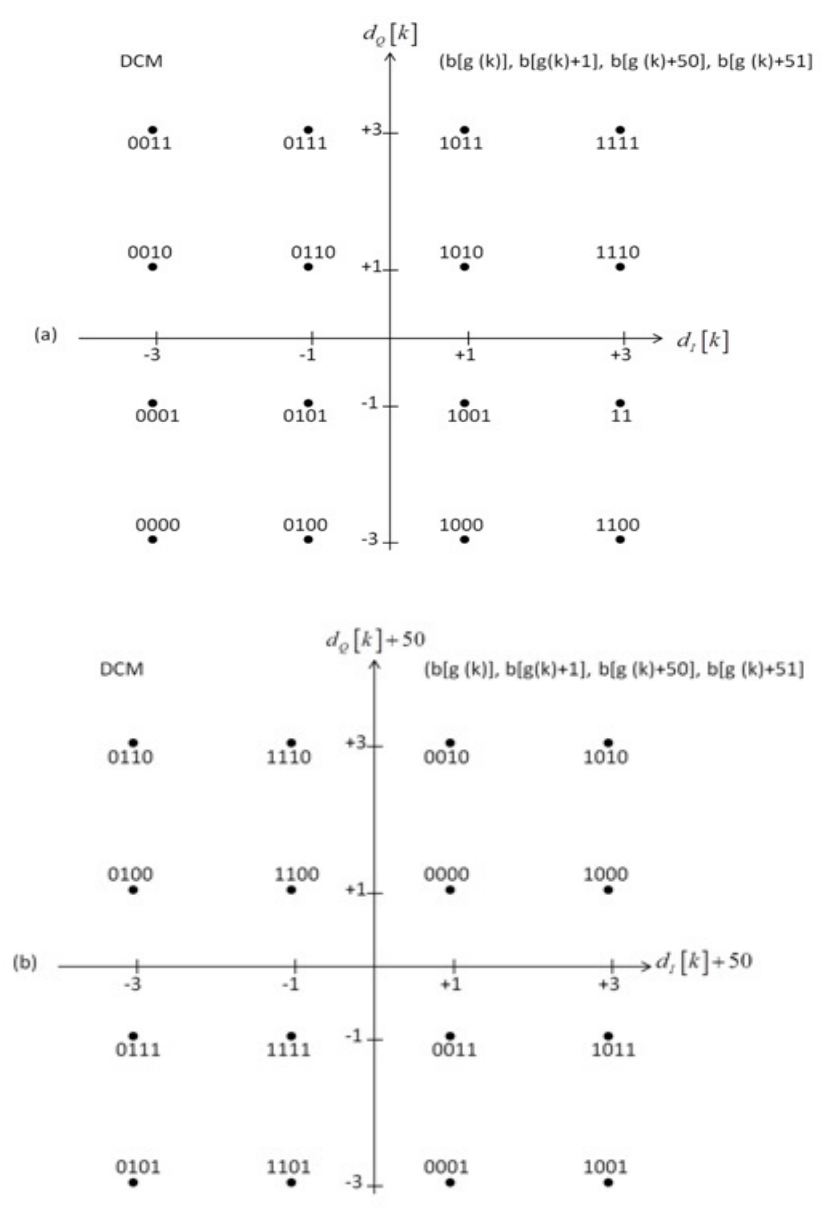

Figure 2: 16-QAM Constellations of the DCM modulation for MB-OFDM system. 
Tableau 2: Encoding table of the dual carrier modulation (DCM).

\begin{tabular}{c|cccc}
\hline \hline $\begin{array}{c}\text { Input bit } \\
(b[g(k)], b[g(k)+1], b[g(k)+50)], b[g(k)+51])\end{array}$ & $\mathrm{D}[\mathrm{k}]$ & $\mathrm{D}[\mathrm{k}]$ & $\mathrm{D}[\mathrm{k}+\mathbf{5 0}]$ & \multicolumn{2}{c}{$\mathrm{D}[\mathrm{k}+\mathbf{5 0}]$} \\
& $\mathrm{I}$-out & Q-out & $\mathrm{I}$-out & Q-out \\
\hline 0000 & -3 & -3 & 1 & 1 \\
0001 & -3 & -1 & 1 & -3 \\
0010 & -3 & 1 & 1 & 3 \\
0011 & -3 & 3 & 1 & -1 \\
0100 & -1 & -3 & -3 & 1 \\
0101 & -1 & -1 & -3 & -3 \\
0110 & -1 & 1 & -3 & 3 \\
0111 & -1 & 3 & -3 & -1 \\
1000 & 1 & -3 & 3 & 1 \\
1001 & 1 & -1 & 3 & -3 \\
1010 & 1 & 1 & 3 & 3 \\
1011 & 1 & 3 & 3 & -1 \\
1100 & 3 & -3 & -1 & 1 \\
1101 & 3 & -1 & -1 & -3 \\
1110 & 3 & 1 & -1 & 3 \\
1111 & 3 & 3 & -1 & -1 \\
\hline \hline
\end{tabular}

\section{SiMULATION RESULTS}

\subsection{Parameters}

In this section, we present some simulations results per-formed on the MB-OFDM system described above in or-der to analyse its performance.

In these simulations, we consider an indoor environment.

In the following, the performance evaluation of the MB-OFDM system is achieved by simulating the transmission chain for the CM1 channel model (channel models), adopted by the working group IEEE 802.15.3a, whose characteristics are shown in Table 3.

The results, expressed in terms of bit error rate (BER), were averaged over 100 iterations of distinct channels of each model CM. Each new transmitted frame of OFDM symbols employs a new channel realization. The stop conditions being themselves tested every 100 received frames, i.e., until all the channels achievements are proven.

The made TFC are those of group 1 (Tab. 4). Thus, the system works using the US regulations set by the FCC. 
The simulation results are evaluated in terms of $\mathrm{Eb} / \mathrm{No}$, where Eb is the average energy per useful bit and No is the power density of AWGN (Additive white Gaussian noise), and perfect channel estimation is considered.

The performance of the MB-OFDM system is shown for various flow rates listed in Table 5. Note that the parameters considered here are those adopted in [5].

Replacing QPSK by DCM slightly improves the system performance to the detriment of the complexity of the higher-level system.

In reception, the used equalization technique is $\mathrm{ZF}$. It is implemented from the coefficients obtained through a full assessment of the transmission channel.

Tableau 1: IEEE 802.15.3a Model Specifications for four distinct configurations.

\begin{tabular}{l|llll}
\hline \hline & CM1 & CM2 & CM3 & CM4 \\
\hline $\boldsymbol{\tau}_{\mathrm{m}}$, Mean execs delay (ns) & 5.05 & 10.38 & 14.18 & 27 \\
$\boldsymbol{\tau}_{\mathrm{RMS}}, \mathrm{RMS}$ (root -Mean-square) delay speed (ns) & 5.28 & 8.03 & 14.28 & 25 \\
$N P_{10 \mathrm{aB}}^{(a)}$ & 13 & 18 & 35 & 41 \\
$N P_{85 \%}^{(b)}$ & 24 & 36.1 & 61.54 & 123 \\
\hline \hline
\end{tabular}

(a) Number of paths above the threshold of $-10 \mathrm{~dB}$ relative to the dominant path

(b) Number of paths containing $85 \%$ of the energy of the impulse response

Tableau 2: Time-Frequency codes (TFC) for the band group 1 [4].

\begin{tabular}{l|lc}
\hline \hline \multicolumn{1}{c|}{ TFC Number } & \multicolumn{1}{|c}{ TFC type } & TFC code \\
\hline $\mathbf{1}$ & TFI & 123123 \\
$\mathbf{2}$ & & 132132 \\
$\mathbf{3}$ & & 112233 \\
$\mathbf{4}$ & FFI & 113322 \\
$\mathbf{5}$ & & 111111 \\
$\mathbf{6}$ & & 222222 \\
$\mathbf{7}$ & TFI2 (TFI over 2 sub- & 333333 \\
$\mathbf{8}$ & bands) & 121212 \\
$\mathbf{9}$ & & 231313 \\
$\mathbf{1 0}$ & & 23123 \\
\hline \hline
\end{tabular}


Tableau 3: Rates of MB-OFDM system based on the ECMA-368 standard [4].

\begin{tabular}{l|llllll}
\hline \hline $\begin{array}{l}\text { Rate } \\
\text { (Mbit/s) }\end{array}$ & Modulation & $\begin{array}{l}\text { Coding } \\
\text { Efficiency } \\
(\boldsymbol{R})\end{array}$ & FDS & TDS & $\begin{array}{l}\text { Coded bits } \\
\text { per symbol }\end{array}$ & $\begin{array}{l}\text { useful bits per } \\
\text { block }\end{array}$ \\
\hline $\mathbf{5 3 3}$ & QPSK & $1 / 3$ & Yes & Yes & 200 & 100 \\
$\mathbf{8 0}$ & QPSK & $1 / 2$ & Yes & Yes & 200 & 150 \\
$\mathbf{1 0 6 . 7}$ & QPSK & $1 / 3$ & No & Yes & 200 & 200 \\
$\mathbf{1 6 0}$ & QPSK & $1 / 2$ & No & Yes & 200 & 300 \\
$\mathbf{2 0 0}$ & QPSK & $5 / 8$ & No & Yes & 200 & 375 \\
$\mathbf{3 2 0}$ & DCM & $1 / 2$ & No & No & 200 & 600 \\
$\mathbf{4 0 0}$ & DCM & $5 / 8$ & No & No & 200 & 750 \\
$\mathbf{4 8 0}$ & DCM & $3 / 4$ & No & No & 200 & 900 \\
\hline \hline
\end{tabular}

\subsection{DCM vs QPSK}

Dual carrier modulation (DCM) is being studied as a potential improvement of the classic modulation QPSK. DCM provides not only a diversity gain, but also a coding gain. DCM results are compared with those using conventional QPSK.

Therefore, the simulation results show that the DCM achieves lower BER compared to conventional QPSK.

Figure 4 shows that for a BER value of 10-3 a gain of about $0.7 \mathrm{~dB}$ is observed with a $320 \mathrm{Mbit} / \mathrm{s}$ throughput for both cases with or without TFC (Time Frequency Code).

According to these results, the DCM method is more efficient than QPSK. The advantages of DCM are retained even when the channel coding is applied.

The use of DCM provides the selection of an increased return code. DCM is not only robust, but also offers improvements in speed for a signal to noise ratio (SNR (signal to noise ratio)) given.

This improvement in performance is related to the characteristics of DCM, which provides additional diversity gain, first by spreading the symbol on two independent sub-carriers and secondly by the use of coding gain with constellation rearrangement. 


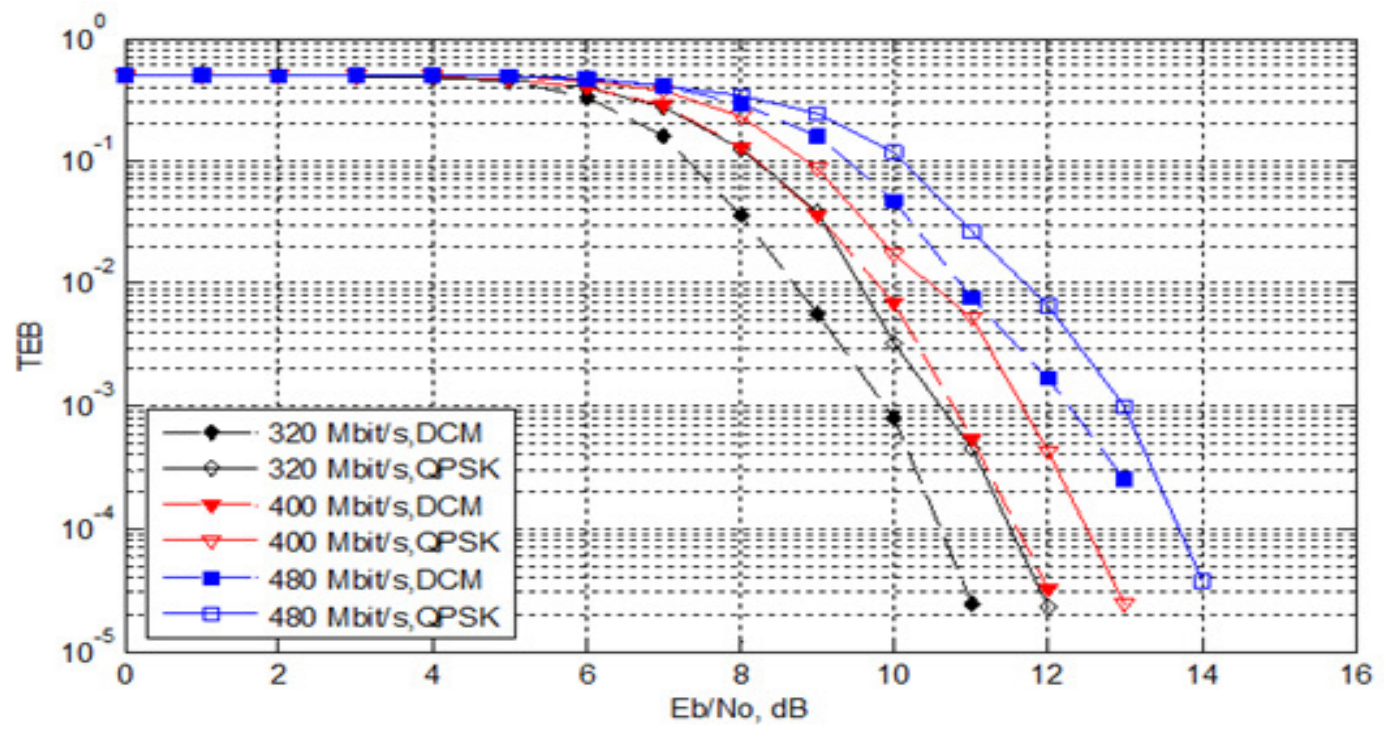

(a) Without TFC

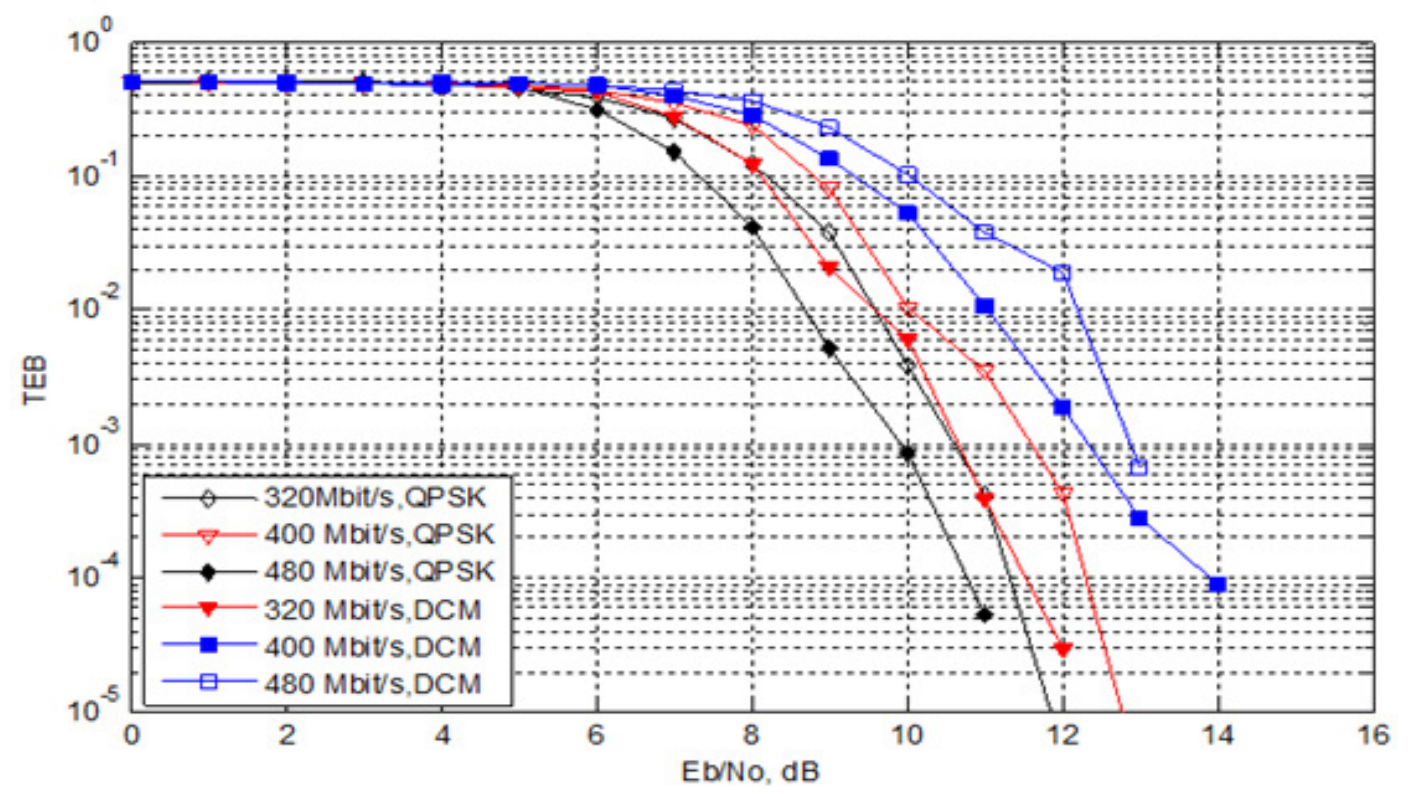

(b) With TFC

Figure 3: DCM versus QPSK for high data rates of $320 \mathrm{Mb} / \mathrm{s}, 400 \mathrm{Mb} / \mathrm{s}$ and $480 \mathrm{Mb} / \mathrm{s}$ Simulation in the case of CM1 (LOS) without TFC n ${ }^{\circ} 5$ and with TFC n ${ }^{\circ} 1$. 


\section{CONCLUSION}

In this paper, a performance comparison between DCM and QPSK modulation was evaluated in a UWB system MBOFDM.

This evaluation shows the importance of using the new technology DCM only for high flow rates for both cases with and without TFC in MBOFDM system. Therefore, QPSK modulation is replaced by a multidimensional modulation DCM.

\section{REFERENCES}

[1] Federal Communication Commission, Revision of Part 15 of the Commission's Rules Regarding Ultra-Wideband Transmission Systems, First Report and Order, ET Docket 98-153, FCC 02-48, Feb. 2002.

[2] D. Porcino and W. Hirt, "Ultra-wideband radio technology: Potential and challenges ahead, " IEEE Communication Magazine, vol. 41, pp.66-74, 2003.

[3] "MultiBand OFDM Physical Layer Specification Release 1.0," 2005

[4] ECMA, "High rateUltra Wideband PHY and MAC Standard “. Rapport ECMA-368 3nd edition, ECMA International, Décembre 2008.

[5] A. Batra et al., "Multi-band OFDM Physical Layer Proposal for IEEE 802.15 Task Group 3a ". IEEE P802.15-04/0493r1, Septembre 2004.

[6] G. F. Ross, "Transmission and reception system for generating and receiving base-band duration pulse signals for short base-band pulse communication system“, U.S. Patent 3,728,025 dated July 31, 1973.

[7] M. Hajjaj, Walid Chainbi, Ridha Bouallegue, "A Rhombic-DCM Constellation for MB-OFDM UWB Systems ",IEEE International Wireless Communica-tions \& Mobile Computing (IWCMC), Dubrovnik ,p.256 - 261, Aug. 2015. 